\title{
F. CHAUNY
}

M. CHOKRON

A. HAURIE

\section{Prévision de la demande de soins à partir d'un système d'information décentralisé}

Revue française d'automatique, d'informatique et de recherche opérationnelle. Recherche opérationnelle, tome 18, n 2 (1984), p. 173-194.

$<$ http://www.numdam.org/item?id=RO_1984_18_2_173_0>

(C) AFCET, 1984, tous droits réservés.

L'accès aux archives de la revue « Revue française d'automatique, d'informatique et de recherche opérationnelle. Recherche opérationnelle » implique l'accord avec les conditions générales d'utilisation (http://www.numdam.org/ legal.php). Toute utilisation commerciale ou impression systématique est constitutive d'une infraction pénale. Toute copie ou impression de ce fichier doit contenir la présente mention de copyright.

\section{Numdam}

Article numérisé dans le cadre du programme

Numérisation de documents anciens mathématiques

http://www.numdam.org/ 


\title{
PRÉVISION DE LA DEMANDE DE SOINS A PARTIR D'UN SYSTEMME D'INFORMATION DÉCENTRALISÉ (*)
}

\author{
par F. Chauny, M. Chokron et A. Haurie $\left({ }^{1}\right)$
}

\begin{abstract}
Résumé. - Cet article présente une méthodologie de prévision de la demande de soins infirmiers dans un service hospitalier et son implantation sur un système informatique décentralisé (microordinateur). La prévision est obtenue à partir d'une identification de la structure dynamique de base qui décrit lévolution des conditions de maladie des patients hospitalisés. La dynamique des maladies est ainsi représentée par des processus semi-markoviens à états discrets. A chaque état est associée une loi de demande de soins typiques. Connaissant le recensement des patients dans Tunité, un jour donné, on peut alors prévoir lévolution de la demande de soins pour les jours à venir.
\end{abstract}

Le système a été expérimenté dans une unité de gynécologie d'un hôpital général de Montréal et sa capacité prévisionnelle a été évaluée.

Mots clés : Processus semi-Markoviens; prévision de la demande de soins; système d'information décentralisé.

Abstract. - This paper deals with a prediction model for the nursing care demand in a surgical department. The model is based on a description of disease dynamics by semi-Markov stochastic processes and on the identification of typical care requirements associated with each state of disease. The prediction model has been implemented on a decentralized information system using a small desk computer, and the methodology has been tested in both a simulated and real environments.

The results show a good predictive capability on an horizon of several days. This should permit the nursing care managers to improve their resource allocation.

Keywords: Stochastic modeling; Semi-Markov processes; health care; decentralized information system.

\section{INTRODUCTION}

Le salaire du personnel infirmier est le principal poste budgétaire des hôpitaux du Québec. La qualité des soins est d'autre part très étroitement reliée à la capacité du personnel infirmier à satisfaire la demande de soins provenant des patients hospitalisés. La gestion du personnel infirmier est donc

(*) Reçu en avril 1983.

Cette recherche a bénéficié du soutien financier du C.R.S.N.G. et du C.R.S.H. (Canada), du fonds F.C.A.C., du fonds de développement et de l'Alma Mater de l'Université de Montréal ainsi que du service à la recherche de l'École des H.E.C.

( ${ }^{1}$ ) GERAD, Ecole des Hautes Études Commerciales, 5255, avenue Decelles, Montréal, Québec H3T 1V6, Canada. 
particulièrement importante et a suscité déjà plusieurs études de recherche opérationnelle. Nous pourrions séparer ces études en deux grandes catégories : celles qui ont trait aux politiques d'affectation du personnel (ex. $[9,16,18]$, etc.) et celles qui ont trait aux mesures et évaluations de la charge de travail pour le personnel infirmier (ex. [11, 13, 17], etc.). Dans cette seconde catégorie le projet PRN [15] mérite une attention spéciale. Le système d'évaluation développé dans ce projet a été utilisé dans plusieurs hôpitaux canadiens et il a permis de recueillir des statistiques utiles sur l'évolution des charges de travail dans différentes unités de soins. Ces statistiques, relevées sur une longue période, permettent à la direction de l'hôpital de constater a posteriori ce qui s'est passé au niveau des soins infirmiers et de prendre des mesures correctives pour l'avenir. L'élément central de PRN est une formule d'évaluation de la demande de soins qui est directement associée au formulaire de plan de soins que chaque infirmière doit remplir chaque jour, pour chaque patient.

Si le système d'évaluation PRN fournit un outil de gestion très utile pour la direction d'un hôpital, il peut néanmoins ne pas être perçu de façon très positive par le personnel infirmier directement concerné. En effet le système est basé sur un système d'information centralisé qui peut être interprété comme une sorte de système de surveillance. De plus, le système dans son état actuel ne permet pas de prévoir les fluctuations de charge de travail à court terme; or c'est dans l'élimination des pointes de demandes que réside l'amélioration de la qualité des soins et la réduction du coût.

Dans une recherche préliminaire $[5,6]$ il a été possible de montrer qu'une prévision de la demande de soins infirmiers pouvait être obtenue à partir de l'identification de la "dynamique de maladie » des patients traités dans une unité de soins. On a pu montrer aussi que de telles prévisions pouvaient être utiles pour réaliser une régulation de la demande de soins par un contrôle sélectif des admissions ainsi qu'une régulation de l'offre de soins par une politique d'utilisation du " personnel volant ». Cette recherche souffrait cependant de deux limitations: tout d'abord elle était basée sur une description très succinte de la variété des maladies traitées et, surtout, elle restait dépendante d'un système d'information centralisé qui pouvait, pour des raisons d'ordre psychologique ou syndicale, établir un obstacle entre le système et ceux qui devraient en être les principaux bénéficiaires.

Les progrès récents de la micro-informatique nous ont permis de réaliser un système d'information totalement décentralisé qui donne à une infirmière chef la possibilité de prévoir quelques jours à l'avance l'évolution de l'état de son unité. Le système est entièrement autonome, implanté sur un ordinateur HP9835, de $64 \mathrm{~K}$ de mémoires, avec deux disquettes et doté d'une capacité 
conversationnelle (bilingue) qui en rend l'usage facile sans formation préalable. Une description du système informatique est donnée dans [2] et [4].

Dans cet article nous analyserons les résultats obtenus après une période test de plusieurs mois dans une unité de gynécologie d'un hôpital général de Montréal. Notre but est donc d'évaluer la capacité de prévision que fournit un tel système, à partir d'une utilisation en site réel.

En section 2 nous présenterons la méthodologie de prévision qui est fondamentalement basée sur une description de la dynamique des maladies à l'aide de processus semi-markoviens.

Nous donnerons, en section 3, une brève description du système tel qu'implanté sur micro-ordinateur.

En section 4, nous traiterons de notre expérience en milieu hospitalier. Nous présenterons d'abord le contexte dans lequel fût placé le microordinateur. Nous verrons ensuite comment sont estimés les paramètres de base du modèle. Nous évaluerons enfin les capacités prévisionnelles du système. Cette évaluation se sépare en deux volets :

La première analyse consiste en une simulation, réalisée à partir des données de base décrivant les dynamiques de maladie et la demande de soins. La seconde analyse traitera des observations recueillies en site réel.

Cette analyse en deux temps permet de séparer l'effet de l'incertitude inhérente à la dynamique des maladies (processus stochastique) de celui des aléas statistiques associés à l'identification des paramètres du modèle.

En conclusion, nous dégagerons un certain nombre d'implications sur les nouveaux développements à réaliser sur un tel système, pour le rendre encore plus adapté à sa mission.

2. PRÉVISION DE LA DEMANDE DE SOINS A L'AIDE D'UN MOdÈle DE POPULATION SEMI-MARKOVIENNE

Dans cette section nous présentons la problématique générale de la prévision de la demande de soins et la méthodologie adoptée qui est basée sur une description de la "dynamique de maladie» des patients traités. Nous présenterons ensuite les formules mathématiques de prévisions obtenues selon les différentes politiques d'admission envisagées.

\subsection{Problématique de la prévision de la demande de soins}

La demande de soins infirmiers dans une unité de soins peut subir des variations importantes du fait des modifications de la composition de la 
population de patients présents dans l'unité. Les patients peuvent avoir différentes maladies et, dans chacune d'elle, peuvent se trouver dans différents états. Suivant l'état, les médecins prescrivent des soins appropriés qui contribuent à la demande de soins infirmiers.

La demande de soins infirmiers peut donc être considérée comme une quantité déterminée par l'état de l'unité de soins. L'unité elle même est, en fait, une population finie de patients dont les états de maladie évoluent.

Pour modéliser le processus générateur de la demande de soins il faut donc identifier les principales maladies traitées dans l'unité, identifier les différents états de maladie, identifier pour chaque état la demande de soins associée.

Si on connaît les caractéristiques dynamiques de chaque maladie, c'est-àdire la loi d'évolution des états, on peut alors établir une méthodologie de prévision de la demande de soins. L'avantage de cette approche réside dans son caractère structurel. Une modification dans le type de maladies traitées ou dans le type de traitement peut être immédiatement prise en compte dans la méthode de prévision.

Smallwood et al. [14] ont, les premiers, proposé de décrire les dynamiques de maladies à l'aide de processus semi-markoviens. L'idée a été reprise par Kao $[10,11]$ pour la planification d'hôpitaux à soins progressifs et pour la modélisation des maladies coronariennes. Collart et Haurie $[5,6]$ ont adapté le modèle de Kao pour établir un système de commande en "boucle ouverte adaptée » du processus d'admission de patients électifs.

Nous avons retenu, dans cette recherche, le même modèle fondamental de population semi-markovienne pour décrire l'unité de soins. Les paramètres du modèle sont les suivants :

$M$, ensemble de maladies traitées dans l'unité;

$S_{\mu}, \quad$ ensemble des états de la maladie $\mu \in M$;

$p_{i j}^{\mu}$, probabilité de transition de l'état $i \in S_{\mu}$ vers l'état $j \in S_{\mu}$ d'un patient ayant la maladie $\mu$;

$h_{i j}^{\mu}(n)$, probabilité conditionnelle d'un séjour de $n$ jours dans l'état $i$ sachant qu'une transition de $i$ vers $j$ doit avoir lieu;

$\pi_{j}^{\mu}(1)$, probabilité qu'un patient urgent arrivé avec la maladie $\mu$ soit dans l'état $j$;

$\theta_{j}^{\mu}(1)$, probabilité similaire pour un patient électif;

$\beta_{\mu}, \quad$ probabilité pour un patient électif d'avoir la maladie $\mu$;

$u_{\mu}(x)$, probabilité qu'il arrive $x$ patients urgents atteints de la maladie $\mu$, en un jour fixé.

Si l'unité compte $B$ lits, elle est alors représentée par une population de processus semi-markoviens, de taille bornée. 
Le personnel infirmier est réparti en trois équipes (matin, soir, nuit). Soit $d_{j q}^{\mu}$ la variable aléatoire qui représente la charge de travail pour l'équipe $q$, évaluée en minutes, associée à un patient qui se trouve dans l'état $j$ de la maladie $\mu$.

\subsection{Prévision de la demande pour les patients présents et pour les patients urgents qui seront admis}

Nous allons considérer une suite de jours $t, t+1, \ldots, t+\Delta$. Nous distinguerons trois flux de patients. Au jour $t+\delta$ un patient de l'unité sera ou bien un patient qui était déjà présent dans l'unité au jour $t$, ou bien un patient admis en $t+1, \ldots, t+\delta$ à titre d'urgent ou d'électif. Nous présenterons d'abord les formules de prévision relatives aux patients présents en $t$ et aux patients urgents.

\subsubsection{Les patients présents en $t$.}

Soit un patient $\alpha$ présent dans l'unité au jour $t$ et qui se trouve dans l'état $i_{\alpha}$ de la maladie $\mu$ depuis $r_{\alpha}$ jours. A partir des données de base de la dynamique de la maladie $\mu$ on peut calculer ( $c f$. [5]), la probabilité :

$\psi_{j}^{\mu}\left(\delta / i_{\alpha}, r_{\alpha}\right)$, probabilité de trouver en $t+\delta$ dans l'état $j \in S_{\mu}$ un patient qui se trouvait en $t$ dans l'état $i_{\alpha} \in S_{\mu}$ depuis $r_{\alpha}$ jours.

La demande de soins pour l'équipe $q$ associée à ce patient $\alpha$ au jour $t+\delta$ sera alors une variable aléatoire $W_{\alpha q}(\delta)$ qui vérifiera :

$$
E\left[W_{\alpha q}(\delta)\right]=\sum_{j \in S_{\mu}} \psi_{j}^{\mu}\left(\delta / i_{\alpha}, r_{\alpha}\right) E\left[d_{j q}^{\mu}\right]
$$

et :

$$
\operatorname{Var}\left[W_{\alpha q}(\delta)\right]=\sum_{j \in S_{\mu}} \psi_{j}^{\mu}\left(\delta / i_{\alpha}, r_{\alpha}\right)\left(\operatorname{Var}\left[d_{j q}^{\mu}\right]+\left(E\left[d_{j q}^{\mu}\right]-E\left[W_{\alpha q}(\delta)\right]\right)^{2}\right)
$$

Puisque chaque patient évolue indépendament des autres, la charge de travail due aux patients présents en $t$ sera la variable aléatoire:

$$
W_{P q}(\delta)=\sum_{\alpha \in P} W_{\alpha q}(\delta),
$$

pour laquelle :

$$
\begin{gathered}
E\left[W_{P q}(\delta)\right]=\sum_{\alpha \in P} E\left[W_{\alpha q}(\delta)\right], \\
\operatorname{Var}\left[W_{P q}(\delta)\right]=\sum_{\alpha \in P} \operatorname{Var}\left[W_{\alpha q}(\delta)\right] .
\end{gathered}
$$

où $P$ est l'ensemble des patients présents au jour $t$. 
Parmi les états de la maladie $\mu$, certains sont des états de congé. Un patient qui entre dans cet état quitte l'unité le jour même, sans engendrer de demande de soins. Soit $F_{\mu}$ l'ensemble des états de congé de la maladie $\mu$.

Soit $N_{p}(\delta)$ la variable aléatoire qui donne le nombre de patients présents au temps $t$ qui le seront encore au jour $t+\delta$. On aura alors :

$$
E\left[N_{P}(\delta)\right]=N_{P}(0)-\sum_{\alpha \in P} \sum_{e \in F_{\mu}} \psi_{e}^{\mu}\left(\delta / i_{\alpha}, r_{\alpha}\right)
$$

\subsubsection{Les patients urgents admis après le jour $t$}

Soit un patient $\alpha$ qui est admis dans l'unité de soins à titre d'urgent au jour 1. A partir des données de base de la dynamique de la maladie $\mu$ on peut calculer ( $c f .[5])$, la probabilité :

$\Pi_{j}^{\mu}(\tau)$, probabilité de trouver en $t+\tau$, dans l'état $j \in S_{\mu}$, un patient urgent admis au jour $t+1$ avec la maladie $\mu$.

Notons $W_{\alpha q}^{u r \mu}(\tau, \delta)$ la variable aléatoire qui décrit la demande de soins pour l'équipe $q$ du jour $t+\delta$, provenant d'un patient urgent admis le jour $t+\tau,(\delta \geqq \tau)$.

On aura alors :

$$
E\left[W_{\alpha q}^{u r \mu}(\tau, \delta)\right]=\sum_{j \in S_{\mu}} E\left[d_{j q}^{\mu}\right] \Pi_{j}^{\mu}(\delta-\tau+1),
$$

$$
\begin{aligned}
\operatorname{Var}\left[W_{\alpha q}^{u r \mu}(\tau, \delta)\right]=\sum_{j \in S_{\mu}} \Pi_{j}^{\mu}(\delta-\tau+1)\left[\operatorname{Var}\left(d_{j q}^{\mu}\right)\right. \\
\\
\left.+\left\{E\left(d_{j q}^{\mu}\right)-E\left(W_{\alpha q}^{u r \mu}(\tau, \delta)\right)\right\}^{2}\right] .
\end{aligned}
$$

Soit $e_{\mu}(\tau)$ la variable aléatoire qui donne le nombre de patients urgents admis le jour $t+\tau$ avec la maladie $\mu$. On supposera que les variables aléatoires $e_{\mu}(\tau)$ et $e_{\mu^{\prime}}\left(\tau^{\prime}\right)$ sont indépendantes si $\mu \neq \mu^{\prime}$ ou $\tau \neq \tau^{\prime}$.

Soit $W_{q}^{u r \mu}(\tau, \delta)$ la demande de soins qui s'adresse à l'équipe $q$ du jour $t+\delta$ et qui provient des patients urgents admis le jour $t+\tau$ avec la maladie $\mu$.

On aura alors :

$$
E\left[W_{q}^{u r \mu}(\tau, \delta)\right]=E\left[e_{\mu}(\tau)\right] . E\left[W_{\alpha q}^{u r \mu}(\tau, \delta)\right]
$$

$$
\begin{aligned}
\operatorname{Var}\left[W_{q}^{u r \mu}(\tau, \delta)\right]=E\left[e_{\mu}(\tau)\right] . & \operatorname{Var}\left(W_{\alpha q}^{u r \mu}(\tau, \delta)\right) \\
& +\operatorname{Var}\left[e_{\mu}(\tau)\right] \cdot\left(E\left[W_{\alpha q}^{u r \mu}(\tau, \delta)\right]\right)^{2}
\end{aligned}
$$

Comme les arrivées de patients urgents dans chaque maladie et pour chaque jour sont des variables aléatoires indépendantes on obtient la demande totale 
de soins qui s'adresse à l'équipe $q$ du jour $t+\delta$ et qui provient de patients urgents admis entre les jours $t+1$ et $t+\delta$ comme la somme :

$$
W_{q}^{u r}(\delta)=\sum_{\mu \in M} \sum_{\tau=1}^{\delta} W_{q}^{u r \mu}(\tau, \delta)
$$

avec :

$$
\begin{gathered}
E\left[W_{q}^{u r}(\delta)\right]=\sum_{\mu \in M} \sum_{\tau=1}^{\delta} E\left[W_{q}^{u r \mu}(\tau, \delta)\right], \\
\operatorname{Var}\left(W_{q}^{u r}(\delta)\right)=\sum_{\mu \in M} \sum_{\tau=1}^{\delta} \operatorname{Var}\left[W_{q}^{u r \mu}(\tau, \delta)\right] .
\end{gathered}
$$

Soit $N_{u r}(\delta)$ le nombre de patients urgents arrivés entre les jours $t+1$ et $t+\delta$ inclusivement et qui seront présents au jour $t+\delta$. On aura alors :

$$
E\left[N_{u r}(\delta)\right]=\sum_{\tau=1}^{\delta} \sum_{\mu \in M} \sum_{x=0}^{\infty} x\left(1-\sum_{j \in F_{\mu}} \Pi_{j}^{\mu}(\tau)\right) u_{\mu}(x) .
$$

\subsection{Prévision de la demande de soins due aux admissions de patients électifs.}

On appelle "patient électif » un patient qui ne requiert pas une admission d'urgence et qui peut donc être admis seulement si un lit est disponible ou bien si du personnel est disponible pour le soigner.

Nous considérerons deux cas. Le premier correspond à une politique d'admission dite "en boucle ouverte", selon la terminologie des automaticiens. Dans ce cas on fixe pour les jours $t+1, t+2, \ldots, t+\Delta$ un flux d'admission de patients électifs et on prévoit la demande de soins qu'ils vont engendrer.

Le second cas correspond à une politique «en boucle fermée " où on spécifie que l'unité de soins doit être remplie chaque jour. Dans ce cas le nombre de patients électifs à admettre en $t+\tau$ est aléatoire et dépend des départs de l'unité et des arrivées d'urgents.

\subsubsection{Admission en boucle ouverte}

Soit $a(\tau)$ le nombre, déterminé, d'admission que l'on effectuera au jour $t+\tau, \tau=1,2, \ldots, \Delta$. A partir des données de base on peut calculer ( $c f$. [5]) $\theta_{j}^{\mu}(\tau)$, probabilité qu'un patient électif, admis le jour $t+1$ avec la maladie $\mu$, soit dans l'état $j \in S_{\mu}$ le jour $t+\tau$. 
Soit $W_{q}^{e l}(\delta)$ la demande de soins qui s'adresse à l'équipe $q$ du jour $t+\delta$ et qui provient de patients électifs admis entre les jours $t+1$ et $t+\delta$ inclusivement.

On aura :

$$
E\left[W_{q}^{e l}(\delta)\right]=\sum_{\tau=1}^{\delta} \sum_{\mu \in M} \sum_{j \in S_{\mu}} a(\tau) \beta_{\mu} \theta_{j}^{\mu}(\delta-\tau+1) E\left[d_{j q}^{\mu}\right]
$$

$$
\begin{aligned}
\operatorname{Var}\left[W_{q}^{e l}(\delta)\right]=\sum_{i=1}^{\delta} \sum_{\mu \in M} & \sum_{j \in S_{\mu}} a(\tau) \beta_{\mu} \theta_{j}^{\mu}(\delta-\tau+1)\left(\left[\operatorname{Var}\left[d_{j q}^{\mu}\right]\right.\right. \\
& \left.\left.+\left\{E\left[d_{j q}^{\mu}\right]-\left(\sum_{v \in M} \sum_{i \in S_{v}} E\left[d_{j q}^{v}\right] \beta_{v} \theta_{i}^{v}(\delta-\tau+1)\right)\right\}^{2}\right]\right) .
\end{aligned}
$$

Notons $N_{e l}(\delta)$ le nombre de patients électifs arrivés entre les jours $t+1$ et $t+\delta$ inclusivement qui seront encore présents le jour $t+\delta$. On aura :

$$
E\left[N_{e l}(\delta)\right]=\sum_{\tau=1}^{\delta} \sum_{\mu \in M} a(\tau) \beta_{\mu}\left(1-\sum_{j \in F_{\mu}} \theta_{j}^{\mu}(\delta-\tau+1)\right)
$$

\subsubsection{Admissions en boucle fermée}

Il est pratique de raisonner non plus par patient mais par lit. En effet selon cette politique il y aura toujours un patient par lit. Ainsi définissons : $E_{j}^{v}(\delta)$, probabilité qu'un lit, où un patient a été admis le jour $t+1$, contienne le jour $t+\delta$ un patient dans l'état $j$ de la maladie $v$.

Ces probabilités peuvent s'obtenir par les formules de recurrence suivantes :

et

$$
E_{j}^{v}(1)=\beta_{v} \theta_{j}^{v}(1),
$$

$$
\begin{aligned}
E_{j}^{v}(\delta)=\beta_{v} \theta_{j}^{v}(\delta)+\sum_{\tau=1}^{\delta-1} \sum_{\mu \in M} \sum_{k \in F_{\mu}} \beta_{\mu}\left(\theta_{k}^{\mu}(\tau+1)-\theta_{k}^{\mu}(\tau)\right) E_{j}^{v}(\delta-\tau), & \text { si } \delta=2,3 \ldots
\end{aligned}
$$

On en déduit alors la probabilité :

$\eta_{j}^{v}(\delta / i, \mu, r)$, probabilité qu'un lit soit occupé en $t+\delta$ par un patient se trouvant dans l'état $j$ de la maladie $v$, sachant qu'au jour $t$ ce lit était occupé par un patient qui se trouvait dans l'état $i$ de la maladie $\mu$ depuis $r$ jours,

$$
\begin{aligned}
\eta_{j}^{v}(\delta / i, \mu, r)= & \delta_{v \mu} \psi_{j}^{\mu}(\delta / i, r) \\
& +\sum_{\tau=1}^{\delta}\left\{\sum_{e \in F_{\mu}}\left(\psi_{e}^{\mu}(\tau / i, r)-\psi_{e}^{\mu}(\tau-1 / i, r)\right)\right\} \cdot E_{j}^{v}(\delta-\tau+1),
\end{aligned}
$$

où $\delta_{v \mu}$ est le symbole de Kronecker. 
Soit $W_{\alpha q}(\delta)$ la charge de travail adressée à l'équipe $q$, par le patient qui occupera le lit $\alpha$ au jour $t+\delta$, sachant qu'en $t$ ce lit est occupé par un patient atteint de la maladie $\mu$ et se trouvant dans l'état $i \in S_{\mu}$ depuis $r$ jours.

On obtient alors les formules suivantes pour l'espérance et la variance de $W_{\alpha q}(\delta)$ :

$$
E\left(W_{\alpha q}(\delta)\right)=\sum_{v} \sum_{j \in T_{v}} \eta_{j}^{v}(\delta / i, \mu, r) . E\left(d_{j q}^{v}\right),
$$

$$
\begin{aligned}
\operatorname{Var}\left(W_{\alpha q}(\delta)\right)=\sum_{v} \sum_{j \in T_{v}} \eta_{j}^{v}(\delta / i, \mu, r) \\
\times\left\{\operatorname{Var}\left(d_{j q}^{\vee v}\right)+\left[E\left(d_{j q}^{v}\right)+\left[E\left(d_{j q}^{v}\right)-E\left(W_{\alpha q}(\delta)\right)\right]^{2}\right\},\right.
\end{aligned}
$$

où $T_{\mathrm{v}}=S_{\mathrm{v}}-F_{\mathrm{v}}$.

Du fait de l'indépendance qui existe entre chacun des lits, l'espérance et la variance de la charge totale s'obtiennent par addition sur l'ensemble des lits de l'unité.

\section{MISE EN GEUVRE SUR MICRO-ORDINATEUR}

Le modèle de prévision a été implanté sur un micro-ordinateur HewlettPackard (Modèle HP-9835 A), muni d'une mémoire de $64 \mathrm{~K}$, deux unités de disquette (totalisant 1 megabyte), et d'une imprimante (Modèle HP-2631 A).

Nous ne mentionnons ici que les grandes lignes de l'expérience. Une étude plus détaillée est donnée dans [2 et 4].

Un accent particulier a été mis sur la simplicité d'utilisation (le système s'adresse à des personnes ayant souvent très peu de connaissances informatiques).

Tel que conçu le système demande à une infirmière de mettre à jour les dossiers de patients, chaque fois que cela est nécessaire. Elle peut aussi modifier éventuellement les paramètres régissant l'offre de soins ou le nombre de lits ouverts.

Le système offre alors la possibilité de réaliser des prévisions sur la demande de soins selon un ou plusieurs des trois modes d'admissions suivants.

(i) Admissions d'électifs proposées par l'opérateur.

(ii) Admissions effectuées en fonction d'une pleine occupation de l'unité.

(iii) Admissions basées sur la recherche d'une régulation sous-optimale de la charge de travail (voir [6]). 
La durée d'une séance type (mise à jour des dossiers de patients, prévisions selon deux ou trois modes) est d'environ une demi-heure.

Le système recueille certaines données historiques sur l'évolution de l'état des patients. Les dynamiques des maladies pouvant varier à long terme, ces données permettent d'effectuer une mise à jour des paramètres de base en utilisant éventuellement une procédure bayesienne (voir [7]).

\section{EXPÉRIENCE PILOTE}

\subsection{Le contexte}

Le micro-ordinateur pré-programmé a été installé à l'unité de gynécologie de l'hôpital général Juif de Montréal.

Dans cette unité de gynécologie, pendant l'année 1978, un ensemble restreint de maladies comprenait la majorité des cas (20 diagnostics différents comptaient pour $89,11 \%$ des patients).

On y remarquait aussi un taux d'urgents assez bas (cinq fois moins d'urgents que d'électifs).

\subsection{Données de base}

Les paramètres de base, ont d'abord été évalués lors d'entrevues avec le personnel médical de l'hôpital, puis estimés par la compilation des quelques 1176 dossiers de patients ayant séjourné à l'unité de gynécologie durant l'année 1978.

A la liste des 20 maladies les plus fréquentes, nous ajoutons deux cas, soit "Autres maladies» et "Maladies chroniques». "Autres maladies » correspond à l'ensemble des maladies non répertoriées dans le système, tandis que "Maladies chroniques » correspond à des patients dont l'évolution de l'état se fait à une échelle de temps beaucoup plus longue. La liste des maladies, ainsi formée, apparaît dans le tableau I.

Les entrevues préalables ont été nécessaires pour faire apparaitre les différents états à identifier sur chacun des dossiers consultés ( fig. 1). Les probabilités de transition $p_{i j}^{\mu}$, de séjour $h_{i j}^{\mu}(n)$, de maladie $\beta_{\mu}$, d'état initial $\Pi_{j}^{\mu}(1)$, $\theta_{j}^{\mu}(1)$ ont été déterminées par la méthode du maximum de vraisemblance appliquée aux données recueillies dans les dossiers des patients hospitalisés en 1978. Les probabilités d'arrivées d'urgents $u_{\mu}(x)$, sont issues d'une loi de 
Diagnostic 623.0

Maladie: CYSTOCELE

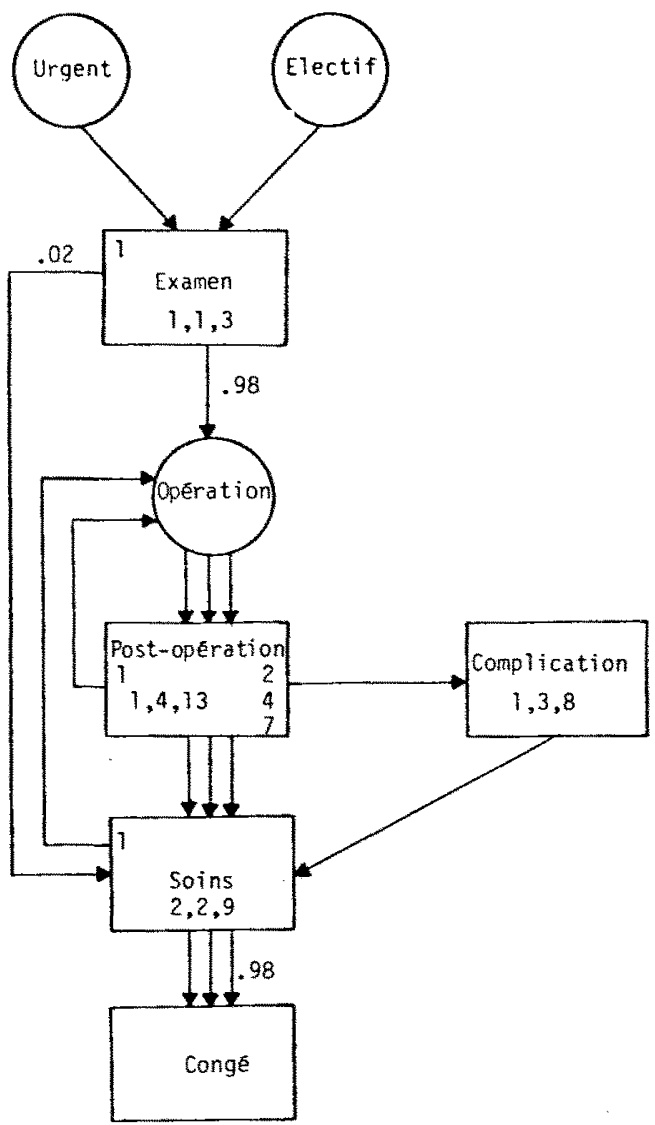

Figure 1. - Arbre représentatif de la dynamique d'une maladie. Les nombres portés sur les arcs représentent les probabilités de transitions. Les nombres associés aux états représentent les durées de séjour minimale, modale et maximale, évaluées en jours.

Poisson de paramètre $e_{\mu}$, estimé par :

$$
\hat{e}_{\mu}=\frac{n_{\mu}}{365}
$$

où $n_{\mu}$ représente le nombre de patients arrivés à l'hôpital, à titre d'urgents, avec la maladie $\mu$, durant l'année 1978 .

L'évaluation des charges de travail consiste à estimer l'espérance et la variance des quantités de soins infirmiers par équipe et par catégorie de personnel, pour chacun des états des 22 maladies retenues. 
TABLEAU I

Liste des maladies

1. Cancer du col utérin.

2. Cancer du corps de l'utérus.

3. Cancer de l'ovaire.

4. Fibrome utérin.

5. Tumeur bénigne de l'ovaire.

6. Kyste ovarien (adénome).

7 Kyste ovarien (rétention).

8. Inflammation pelvienne.

9. Endométriose.

10. Endométriose des annexes (trompe, ovaire).

11. Encerclement du col.

12. Infection de l'utérus (excepté le col).

13. Cystocèle.

14. Rectocèle.

15. Prolapsus utérin.

16. Ménorragie.

17. Métrorragie.

18. Incontinence à l'effort.

19. Bartholinite.

20. Ligature des trompes.

21. Autres maladies.

22. Maladies chroniques.

Cette estimation est réalisée en trois étapes.

Premièrement, à l'aide d'un formulaire PRN 76 (voir [15]), une infirmière établit un plan de soin type pour chacun des états de nos maladies. Chaque état est alors décomposé en actes. Pour chacun de ces actes, l'infirmière évalue, en minutes, les quantités de soins infirmiers minimales, modales et maximales par équipe et par catégorie de personnel. La figure 2 est un exemple d'une telle évaluation.

Deuxièmement, à l'aide d'une méthode interactive, une distribution de Weibull est ajustée, à partir des valeurs totales des quantités, minimales, modales et maximales, de soins infirmiers. Un premier histogramme est imprimé. L'infirmière peut modifier cet histogramme, de manière à ce qu'il reflète plus exactement sa perception des fréquences. Un nouvel histogramme est alors imprimé ( fig. 3).

Troisièmement, à l'aide de l'histogramme final, on détermine la probabilité pour chaque intervalle. On en déduit l'espérance et la variance pour la demande de soins infirmiers par état, pour chaque équipe et chaque catégorie de personnel. Un exemple est donné dans la figure 4. 
Figure 2. - Évaluation des quantités de soins requises pour un état donné.

Diagnostic, 623-0. Maladie, cystocèle. État, examen.

\begin{tabular}{|c|c|c|c|c|c|c|c|c|c|}
\hline Catégorie : Infirmière & \multicolumn{3}{|c|}{ Quart : 1} & \multicolumn{3}{|c|}{ Quart : 2} & \multicolumn{3}{|c|}{ Quart : 3} \\
\hline Plan de soins & $\left({ }^{a}\right)$ & $\left({ }^{b}\right)$ & $\left({ }^{c}\right)$ & $\left({ }^{a}\right)$ & $\left(b^{b}\right)$ & $\left({ }^{c}\right)$ & $\left({ }^{a}\right)$ & $\left(b^{b}\right)$ & $\left({ }^{c}\right)$ \\
\hline Soins d'hygiène & 5 & 5 & 10 & 5 & 5 & 5 & 0 & 0 & 5 \\
\hline Alimentation. & 6 & 6 & 6 & 4 & 4 & 4 & 0 & 0 & 0 \\
\hline Communication Sociale & 0 & 10 & 15 & 0 & 5 & 10 & 0 & 3 & 5 \\
\hline Signes vitaux $\ldots \ldots \ldots$ & 2 & 2 & 2 & 0 & 0 & 2 & 0 & 0 & 2 \\
\hline Pesée et Mensuration . . & 0 & 2 & 5 & 0 & 0 & 5 & 0 & 0 & 0 \\
\hline Surveillance $\ldots \ldots \ldots$ & 0 & 0 & 0 & 0 & 0 & 0 & 5 & 5 & 5 \\
\hline Rasage ..... & 0 & 15 & 15 & 0 & 0 & 15 & 0 & 0 & 0 \\
\hline Collecte Urine . . & 0 & 5 & 5 & 0 & 5 & 5 & 0 & 5 & 5 \\
\hline Prélèvement sang & 0 & 5 & 10 & 0 & 5 & 5 & 0 & 5 & 10 \\
\hline Douche $\ldots \ldots \ldots$ & 0 & 10 & 10 & 0 & 0 & 10 & 0 & 0 & 0 \\
\hline Assistance aux examens & 0 & 10 & 10 & 0 & 10 & 10 & 0 & 0 & 0 \\
\hline Médication $\ldots \ldots \ldots$ & 0 & 0 & 5 & 0 & 2 & 7 & 0 & 2 & 5 \\
\hline Douche vaginal Bétadine & 0 & 10 & 10 & 0 & 0 & 0 & 0 & 0 & 0 \\
\hline TOTAL & 13 & 80 & 103 & 9 & 36 & 78 & 5 & 20 & 37 \\
\hline
\end{tabular}

(a) Min.; ( $\left.{ }^{b}\right)$ Mode; ( $)$ Max.

Figure 3. - Exemple de distribution des soins infirmiers associée à un état de maladie.

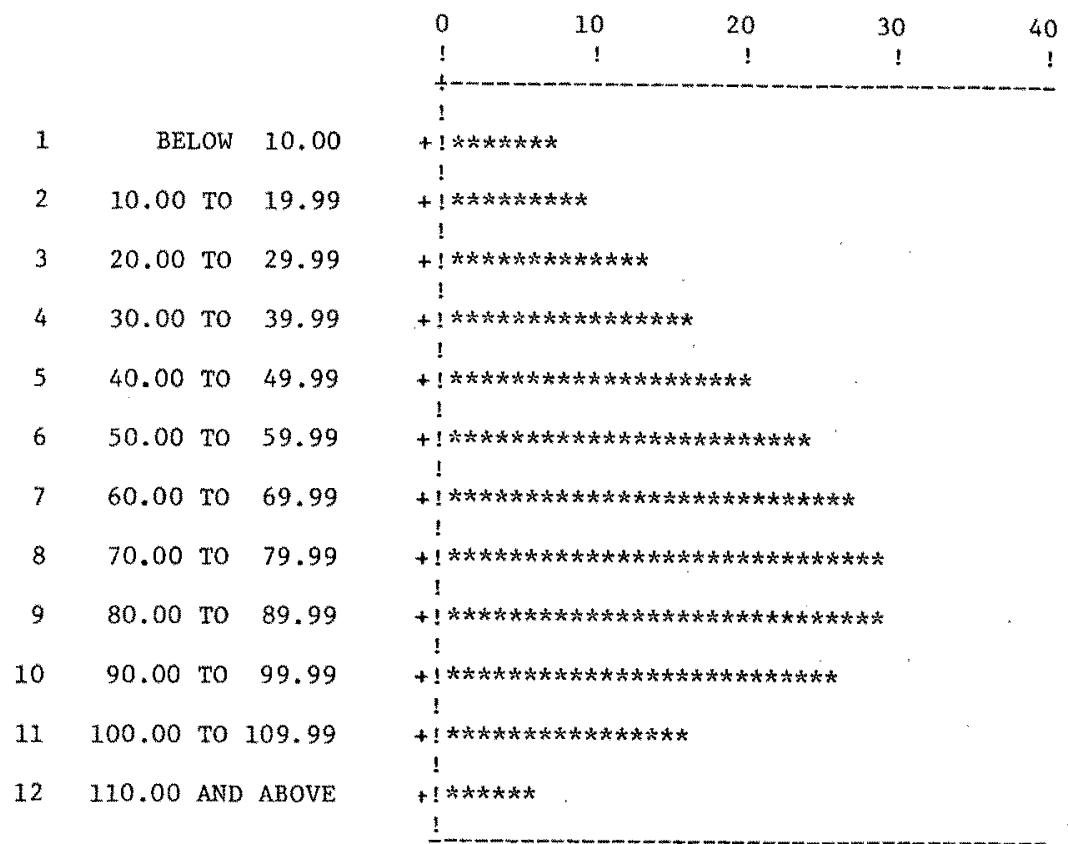

vol. $18, n^{\circ} 2$, mai 1984 
Figure 4. - Quantités de soins infirmiers (minutes).

\begin{tabular}{|c|c|c|c|c|c|c|}
\hline Quart & Examen & Op. & Post-Op. & Compl. & Soins & Congé \\
\hline $\begin{array}{l}\text { Jour : } \\
\text { Moyenne } \\
\text { Variance }\end{array}$ & $\begin{array}{r}65 \\
773\end{array}$ & $\begin{array}{r}53 \\
277\end{array}$ & $\begin{array}{r}78 \\
1219\end{array}$ & $\begin{array}{r}57 \\
426\end{array}$ & $\begin{array}{r}57 \\
657\end{array}$ & $\begin{array}{l}0 \\
0\end{array}$ \\
\hline $\begin{array}{l}\text { Soir : } \\
\text { Moyenne } \\
\text { Variance }\end{array}$ & $\begin{array}{r}44 \\
677\end{array}$ & $\begin{array}{r}69 \\
638\end{array}$ & $\begin{array}{r}69 \\
638\end{array}$ & $\begin{array}{l}52 \\
98\end{array}$ & $\begin{array}{r}44 \\
490\end{array}$ & $\begin{array}{l}0 \\
0\end{array}$ \\
\hline $\begin{array}{l}\text { Nuit : } \\
\text { Moyenne } \\
\text { Variance }\end{array}$ & $\begin{array}{l}18 \\
84\end{array}$ & $\begin{array}{r}38 \\
322\end{array}$ & $\begin{array}{r}38 \\
322\end{array}$ & $\begin{array}{r}35 \\
101\end{array}$ & $\begin{array}{r}20 \\
154\end{array}$ & $\begin{array}{l}0 \\
0\end{array}$ \\
\hline
\end{tabular}

Maladie : Cystocèle (diagnostique 623-0); Catégorie : Infirmière.

\section{3. Évaluation des premiers résultats}

On relève trois causes principales, pour expliquer les différences entre les prévisions et la réalité :

(i) la structure dynamique et stochastique de la population entraîne une incertitude sur l'évolution de la demande de soins;

(ii) les paramètres du modèle sont estimés; de plus, ils sont susceptibles de variation à long terme. Il en résulte une certaine erreur sur les probabilités de base, charges associées aux états, etc.;

(iii) des erreurs de manipulation du système, peuvent se glisser lors de l'entrée des données à court-terme. (Mauvaises prévisions quand au nombre d'admissions, mauvaise définition des états, etc.).

Nous nous intéressons ici aux deux premiers points.

Tout d'abord, afin d'éliminer l'incertitude sur les paramètres, nous évaluerons la capacité prédictive du modèle par voie de simulation. Nous examinerons par la suite, les données relevées à l'hôpital général Juif de Montréal.

Avant d'exposer les résultats, nous allons présenter une description sommaire des données. La méthodologie statistique utilisée sera ensuite décrite. Nous examinerons enfin les principaux résultats obtenus d'abord à partir des données simulées puis à partir des données recueillies à l'hôpital.

\subsubsection{Nature des données}

Pour chaque jour $\delta$ de l'étude, nous possédons le recensement détaillé de l'unité. Nous en déduisons alors : 
(a) Les prévisions de la charge de travail pour les jours $\delta+1$, $\delta+2, \ldots, \delta+7$.

(b) Une charge estimée pour chacune des équipes, au jour $\delta$, obtenue en associant, à chaque patient, la charge espérée $E\left(d_{i q}^{\mu}\right)$, selon son état et sa maladie, et en faisant le total pour l'ensemble des patients.

La simulation nous présentera les capacités de prévision du modèle lorsque l'unité fonctionne à pleine capacité. Ce genre d'analyse a l'avantage d'éliminer toute erreur due à une mauvaise estimation des paramètres. (Les principes de la simulation sont exposés en annexe.)

Les données ont été recueillies à l'hôpital général Juif de Montréal entre le 5 juillet 1981 et le 5 août 1981. Le mois de juillet correspondant à la période de vacance au Québec, se trouve être aussi la saison creuse d'un hôpital. Par conséquent, le taux d'occupation de lits devient instable durant cette période, et nous utiliserons ces données afin d'évaluer les performances du modèle, lorsque le nombre d'admissions d'électifs, pour les jours à venir, est fourni par l'opérateur. Nous avons retiré de l'étude, les patients dont le diagnostic n'apparaît pas dans notre répertoire de 20 maladies. Étant donné la diversité de ces maladies, nous avons jugé préférable d'agir ainsi. Les prévisions ont été recalculées à la fin de l'étude, avec le vrai nombre d'admissions pour les jours à venir, celui-ci étant connu a posteriori. Enfin, disons que la dynamique utilisée pour établir ces prévisions avait été mise à jour à partir des données recueillies entre les mois de janvier et juin 1981.

\subsubsection{Méthodologie statistique}

L'avantage présenté par un tel modèle de prévision, est d'obtenir des prévisions suffisamment proches des charges réelles, pour avoir une variance des erreurs de prévision inférieure à la variance des charges de travail. L'analyse statistique tentera de quantifier :

(1) La proximité entre les charges et leurs prévisions, à l'aide d'un modèle de régression linéaire.

(2) La différence relative entre la variance des erreurs et la variance des charges, avec le rapport de ces deux variances.

Nous avons retenu le modèle de régression.

$$
Y_{q \delta}=\beta_{0 q}(\tau)+\beta_{1 q}(\tau) . P_{q \delta}(\tau)+E_{q \delta}(\tau), \quad \tau=1,2, \ldots, 7,
$$

où :

$Y_{q \delta}$, la charge moyenne, pour l'équipe $q$, pour l'état de l'unité tel qu'observé au jour $\delta$;

$\delta=1,2, \ldots, n, n$, nombre d'observations; 
$P_{q \delta}(\tau)$, la prévision de la charge de soins pour l'équipe $q$, au jour $\delta$, établie selon le recensement de l'unité $\tau$ jours plus tôt;

$\beta_{0 q}(\tau)$ et $\beta_{1 q}(\tau)$, coefficients de régression de $Y_{q \delta}$ sur $P_{q \delta}(\tau)$ (estimés par la méthode des moindres carrés);

$E_{q \delta}(\tau)$, l'erreur due à l'évolution aléatoire de l'état de l'unité.

Nous testerons l'hypothèse suivante :

$$
H_{0}:\left(\begin{array}{c}
\beta_{0 q}(\tau) \\
\beta_{1 q}(\tau)
\end{array}\right)=\left(\begin{array}{l}
0 \\
1
\end{array}\right),
$$

avec la contre hypothèse :

$$
H_{1}:\left(\begin{array}{c}
\beta_{0 q}(\tau) \\
\beta_{1 q}(\tau)
\end{array}\right) \neq\left(\begin{array}{l}
0 \\
1
\end{array}\right),
$$

pour chacune des équipes, et $\tau=1,2, \ldots, 7$.

Nous calculerons les rapports :

$$
\gamma_{q}(\tau)=\frac{S_{q}-V_{q}(\tau)}{S_{q}}, \quad \tau=1,2, \ldots, 7,
$$

où :

$$
S_{q}=\sum_{\alpha}\left(Y_{q \alpha}-\bar{Y}_{q}\right)^{2},
$$

$\bar{Y}_{q}$ étant la moyenne des observations, et :

$$
V_{q}(\tau)=\sum_{\alpha}\left(Y_{q \alpha}-P_{q \delta}(\tau)\right)^{2} .
$$

Ces rapports indiqueront la réduction de la variance due aux prévisions.

\subsubsection{Analyse des données simulées}

Les tableaux II et III présentent les résultats obtenus à partir de 209 simulations réalisées en "boucle fermée". Les colonnes (1) et (2) du tableau II donnent les estimations des coefficients de régression. Les colonnes (3) et (4) indiquent les écart-types associés à ces estimations. La colonne (5) donne la variance résiduelle estimée. Le coefficient d'explication de la variance due à la régression $R^{2}$ est donné dans la colonne (8). Enfin un aperçu du niveau critique apparaît dans la dernière colonne. Le tableau III donne le rapport $\gamma_{q}(\tau)$ par équipe et par jour de prévision.

R.A.I.R.O. Recherche opérationnelle/Operations Research 
TABLEAU II

Étude de régression.

\begin{tabular}{|c|c|c|c|c|c|c|c|c|}
\hline Jour & Quart & $b_{0}$ & $b_{1}$ & $\sqrt{V\left(b_{0}\right)}$ & $\sqrt{V\left(b_{1}\right)}$ & $s^{2}$ & $R^{2}$ & $\begin{array}{l}\text { Niveau } \\
\text { critique }\end{array}$ \\
\hline 1 & $\begin{array}{l}\mathrm{J} \\
\mathrm{S} \\
\mathrm{N}\end{array}$ & $\begin{array}{r}-45.69 \\
34.64 \\
57.93\end{array}$ & $\begin{array}{r}1.029 \\
.971 \\
.926\end{array}$ & $\begin{array}{l}97.63 \\
62.42 \\
39.28\end{array}$ & $\begin{array}{l}.064 \\
.0521 \\
.052\end{array}$ & $\begin{array}{l}2578.95 \\
2400.63 \\
1709.36\end{array}$ & $\begin{array}{l}.55 \\
.63 \\
.60\end{array}$ & $\begin{array}{l}\hat{\alpha}>.25 \\
\hat{\alpha}>.25 \\
\hat{\alpha}=.25\end{array}$ \\
\hline 2 & $\begin{array}{l}\mathbf{J} \\
\mathrm{S} \\
\mathrm{N}\end{array}$ & $\begin{array}{r}-140.44 \\
-151.32 \\
15.79\end{array}$ & $\begin{array}{r}1.090 \\
1.124 \\
.981\end{array}$ & $\begin{array}{r}134.15 \\
109.75 \\
65.90\end{array}$ & $\begin{array}{l}.088 \\
.091 \\
.087\end{array}$ & $\begin{array}{l}3324.41 \\
3716.34 \\
2685.25\end{array}$ & $\begin{array}{l}.43 \\
.42 \\
.38\end{array}$ & $\begin{array}{l}\hat{\alpha}>.25 \\
\hat{\alpha}>.25 \\
\hat{\alpha}>.25\end{array}$ \\
\hline 3 & $\begin{array}{l}\mathrm{J} \\
\mathrm{S} \\
\mathrm{N}\end{array}$ & $\begin{array}{r}-89.23 \\
-215.88 \\
-26.36\end{array}$ & $\begin{array}{l}1.057 \\
1.173 \\
1.028\end{array}$ & $\begin{array}{r}196.16 \\
157.65 \\
93.07\end{array}$ & $\begin{array}{l}.129 \\
.131 \\
.122\end{array}$ & $\begin{array}{l}4366.23 \\
4629.97 \\
3221.89\end{array}$ & $\begin{array}{l}.25 \\
.28 \\
.25\end{array}$ & $\begin{array}{l}\hat{\alpha}>.25 \\
\hat{\alpha}>.10 \\
\hat{\alpha}>.25\end{array}$ \\
\hline 4 & $\begin{array}{l}\mathbf{J} \\
\mathrm{S} \\
\mathbf{N}\end{array}$ & $\begin{array}{r}-34.81 \\
-14.18 \\
0.90\end{array}$ & $\begin{array}{r}1.021 \\
1.002 \\
.986\end{array}$ & $\begin{array}{l}241.03 \\
189.89 \\
112.91\end{array}$ & $\begin{array}{l}.158 \\
.157 \\
.148\end{array}$ & $\begin{array}{l}4817.57 \\
5370.71 \\
3553.51\end{array}$ & $\begin{array}{l}.17 \\
.16 \\
.18\end{array}$ & $\begin{array}{l}\hat{\alpha}>.25 \\
\hat{\alpha}>.05 \\
\hat{\alpha}=.05\end{array}$ \\
\hline 5 & $\begin{array}{l}\mathrm{J} \\
\mathrm{S} \\
\mathrm{N}\end{array}$ & $\begin{array}{r}65.65 \\
-25.70 \\
31.31\end{array}$ & $\begin{array}{r}.956 \\
1.013 \\
.945\end{array}$ & $\begin{array}{l}296.50 \\
221.15 \\
130.87\end{array}$ & $\begin{array}{l}.195 \\
.183 \\
.171\end{array}$ & $\begin{array}{l}5183.52 \\
5599.21 \\
3763.34\end{array}$ & $\begin{array}{l}.10 \\
.13 \\
.13\end{array}$ & $\begin{array}{l}\hat{\alpha}>.25 \\
\hat{\alpha}>.10 \\
\hat{\alpha}>.025\end{array}$ \\
\hline 6 & $\begin{array}{l}\mathbf{J} \\
\mathbf{S} \\
\mathrm{N}\end{array}$ & $\begin{array}{r}-212.89 \\
-255.57 \\
-83.09\end{array}$ & $\begin{array}{l}1.143 \\
1.206 \\
1.092\end{array}$ & $\begin{array}{l}371.30 \\
274.91 \\
158.51\end{array}$ & $\begin{array}{l}.245 \\
.229 \\
.207\end{array}$ & $\begin{array}{l}5235.13 \\
5662.15 \\
3805.40\end{array}$ & $\begin{array}{l}.10 \\
.12 \\
.12\end{array}$ & $\begin{array}{l}\hat{\alpha}>.25 \\
\hat{\alpha}>.25 \\
\hat{\alpha}>.01\end{array}$ \\
\hline 7 & $\begin{array}{l}\mathrm{J} \\
\mathrm{S} \\
\mathrm{N}\end{array}$ & $\begin{array}{r}28.08 \\
-385.35 \\
-210.90\end{array}$ & $\begin{array}{r}.991 \\
1.324 \\
1.262\end{array}$ & $\begin{array}{l}499.13 \\
350.22 \\
208.25\end{array}$ & $\begin{array}{l}.332 \\
.293 \\
.272\end{array}$ & $\begin{array}{l}5546.56 \\
5848.72 \\
3912.57\end{array}$ & $\begin{array}{l}.04 \\
.09 \\
.09\end{array}$ & $\begin{array}{l}\hat{\alpha}=.025 \\
\hat{\alpha}>.25 \\
\hat{\alpha}>.025\end{array}$ \\
\hline
\end{tabular}

\subsubsection{Données recueillies à l'hôpital}

Les tableaux IV et $\mathrm{V}$ présentent les résultats obtenus à partir des données recueillies à l'hôpital général Juif de Montréal.

\subsubsection{Conclusions}

L'examen de ces résultats permet de dégager les conclusions suivantes:

(a) Lorsque les paramètres sont justes (simulation), la variance des erreurs de prévisions est à peu près équivalente à la variance des charges au-delà d'un délai de quatre jours, mais la contribution est très appréciable pour les trois premiers jours. De plus, les niveaux critiques des régressions sont très acceptables sur cette période, ce qui confirme la proximité entre les charges et leurs prévisions.

(b) Les données recueillies à l'hôpital montrent une forte diminution de la variance pour les trois premiers jours. Par contre l'effet est nul les jours suivants. Les régressions montrent qu'on peut considérer les prévisions comme 
TABLEAU III

Réduction de la variance.

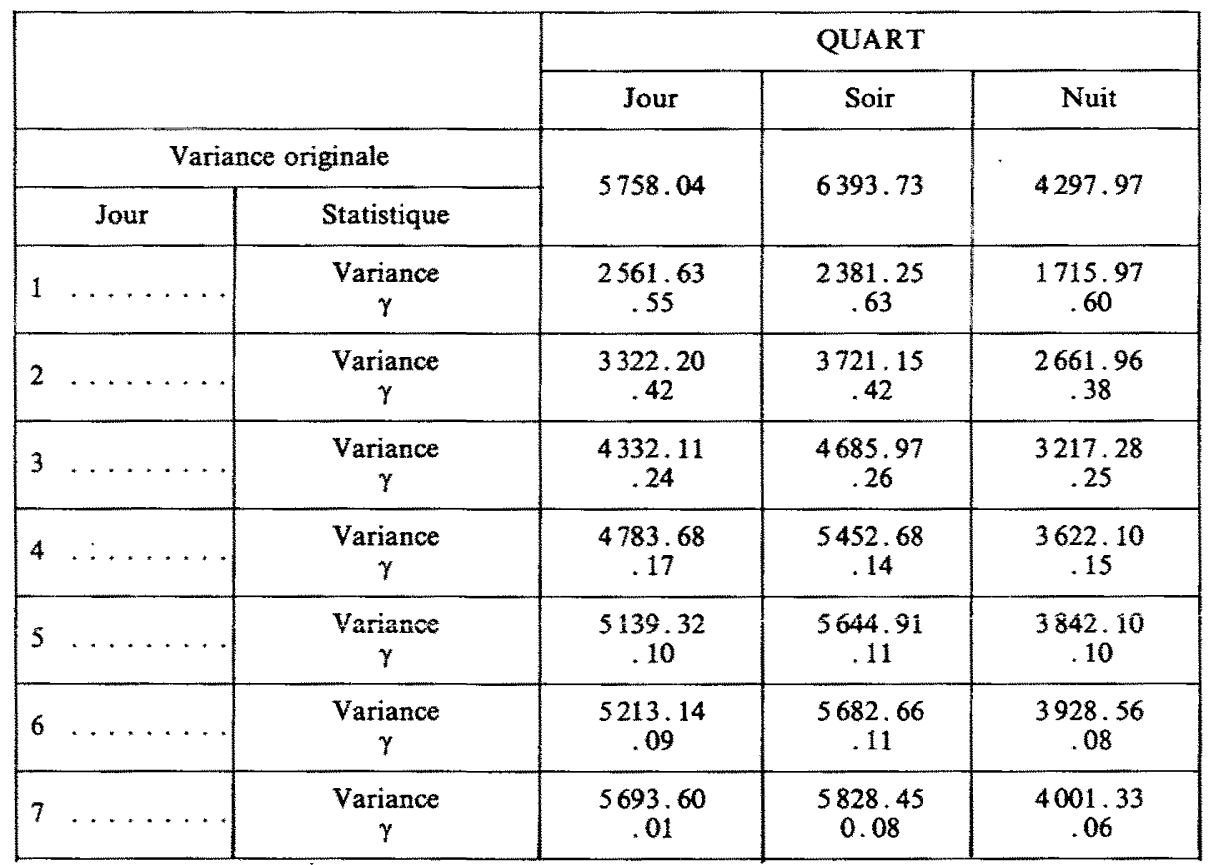

bonnes pour le premier jour, mais leur qualité diminue rapidement ensuite. Nous constatons donc que la capacité théorique de prévision obtenue en ( $a$ ) s'est nettement détériorée quand on considère les prévisions en site réel. Cette détérioration est essentiellement due aux erreurs d'estimation des paramètres des processus semi-Markoviens décrivant la dynamique des maladies. Une réduction de cette incertitude sera obtenue en mettant à jour de façon progressive la banque de données servant à ces estimations.

\section{CONCLUSION}

Les sources de variabilité dans la demande de soins sont très nombreuses. Cependant les résultats obtenus à partir d'une expérience pilote démontrent qu'il est possible de prévoir au moins à court terme (jusqu'à quatre jours à l'avance) l'évolution de la demande de soins totale compte tenu de l'état actuel des patients hospitalisés.

Les implications d'une telle prévision sur la gestion hospitalière peuvent être importantes. En effet la prévision un ou deux jours à l'avance d'une 
TABLEAU IV

Étude de régression sur les données recueillies à l'hôpital.

\begin{tabular}{|c|c|c|c|c|c|c|c|c|}
\hline Jour & Quart & $b_{0}$ & $b_{1}$ & $\sqrt{V\left(b_{0}\right)}$ & $\sqrt{V\left(b_{1}\right)}$ & $s^{2}$ & $R^{2}$ & $\begin{array}{l}\text { Niveau } \\
\text { critique }\end{array}$ \\
\hline 1 & $\begin{array}{l}\mathrm{J} \\
\mathrm{S} \\
\mathrm{N}\end{array}$ & $\begin{array}{r}102.50 \\
57.52 \\
-4.06\end{array}$ & $\begin{array}{l}.84 \\
.91 \\
.98\end{array}$ & $\begin{array}{l}60.14 \\
47.74 \\
30.88\end{array}$ & $\begin{array}{l}.090 \\
.091 \\
.099\end{array}$ & $\begin{array}{l}3980.57 \\
2653.88 \\
1146.32\end{array}$ & $\begin{array}{l}.79 \\
.81 \\
.81\end{array}$ & $\begin{array}{l}\hat{\alpha}>.10 \\
\hat{\alpha}>.25 \\
\hat{\alpha}>.25\end{array}$ \\
\hline 2 & $\begin{array}{l}\mathbf{J} \\
\mathrm{S} \\
\mathrm{N}\end{array}$ & $\begin{array}{l}93.12 \\
43.50 \\
17.16\end{array}$ & $\begin{array}{l}.84 \\
.87 \\
.87\end{array}$ & $\begin{array}{l}65.00 \\
50.62 \\
35.92\end{array}$ & $\begin{array}{l}.096 \\
.094 \\
.110\end{array}$ & $\begin{array}{l}4385.33 \\
2975.45 \\
1619.68\end{array}$ & $\begin{array}{l}.77 \\
.79 \\
.73\end{array}$ & $\begin{array}{l}\hat{\alpha}>.10 \\
\hat{\alpha}>.025 \\
\hat{\alpha}>.01\end{array}$ \\
\hline 3 & $\begin{array}{l}\mathrm{J} \\
\mathrm{S} \\
\mathrm{N}\end{array}$ & $\begin{array}{r}154.20 \\
95.74 \\
64.76\end{array}$ & $\begin{array}{l}.73 \\
.75 \\
.70\end{array}$ & $\begin{array}{l}75.65 \\
63.35 \\
45.64\end{array}$ & $\begin{array}{l}.109 \\
.114 \\
.135\end{array}$ & $\begin{array}{l}6425.38 \\
4898.43 \\
2788.31\end{array}$ & $\begin{array}{l}.66 \\
.65 \\
.54\end{array}$ & $\begin{array}{l}\hat{\alpha}>.01 \\
\hat{\alpha}>.005 \\
\hat{\alpha}=0\end{array}$ \\
\hline 4 & $\begin{array}{l}\mathrm{J} \\
\mathrm{S} \\
\mathbf{N}\end{array}$ & $\begin{array}{r}201.18 \\
129.46 \\
93.90\end{array}$ & $\begin{array}{l}.65 \\
.67 \\
.59\end{array}$ & $\begin{array}{l}78.23 \\
67.39 \\
48.00\end{array}$ & $\begin{array}{l}.109 \\
.118 \\
.138\end{array}$ & $\begin{array}{l}7578.53 \\
5874.34 \\
3340.31\end{array}$ & $\begin{array}{l}.60 \\
.58 \\
.44\end{array}$ & $\begin{array}{l}\hat{\alpha}=0 \\
\hat{\alpha}=0 \\
\hat{\alpha}=0\end{array}$ \\
\hline 5 & $\begin{array}{l}\mathrm{J} \\
\mathbf{S} \\
\mathrm{N}\end{array}$ & $\begin{array}{l}249.20 \\
161.74 \\
110.51\end{array}$ & $\begin{array}{l}.56 \\
.60 \\
.53\end{array}$ & $\begin{array}{l}84.31 \\
72.47 \\
49.45\end{array}$ & $\begin{array}{l}.115 \\
.124 \\
.138\end{array}$ & $\begin{array}{l}9323.28 \\
7008.57 \\
3676.49\end{array}$ & $\begin{array}{l}.51 \\
.50 \\
.39\end{array}$ & $\begin{array}{l}\hat{\alpha}=0 \\
\hat{\alpha}=0 \\
\hat{\alpha}=0\end{array}$ \\
\hline 6 & $\begin{array}{l}J \\
S \\
N\end{array}$ & $\begin{array}{l}263.49 \\
172.29 \\
110.74\end{array}$ & $\begin{array}{l}.53 \\
.57 \\
.52\end{array}$ & $\begin{array}{l}85.18 \\
73.86 \\
49.46\end{array}$ & $\begin{array}{l}.115 \\
.124 \\
.136\end{array}$ & $\begin{array}{l}9825.89 \\
7362.51 \\
3680.72\end{array}$ & $\begin{array}{l}.48 \\
.48 \\
.39\end{array}$ & $\begin{array}{l}\hat{\alpha}=0 \\
\hat{\alpha}=0 \\
\hat{\alpha}=0\end{array}$ \\
\hline 7 & $\begin{array}{l}\mathrm{J} \\
\mathbf{S} \\
\mathbf{N}\end{array}$ & $\begin{array}{l}285.50 \\
185.43 \\
113.24\end{array}$ & $\begin{array}{l}.50 \\
.54 \\
.50\end{array}$ & $\begin{array}{l}85.00 \\
73.13 \\
48.59\end{array}$ & $\begin{array}{l}.112 \\
.122 \\
.132\end{array}$ & $\begin{array}{r}10287.49 \\
7568.11 \\
3666.47\end{array}$ & $\begin{array}{l}.46 \\
.46 \\
.39\end{array}$ & $\begin{array}{l}\hat{\alpha}=0 \\
\hat{\alpha}=0 \\
\hat{\alpha}=0\end{array}$ \\
\hline
\end{tabular}

"surchauffe " de l'unité permet de réquisitionner du personnel supplémentaire ou de transférer des infirmières d'une équipe volante pour atténuer l'impact sur la qualité des soins. D'autre part le système va recueillir au cours de son utilisation quotidienne de plus en plus d'information sur la dynamique des maladies traitées dans l'unité. Cette information va permettre d'adapter les paramètres de base de façon à ce que la description de la dynamique reste fidèle. Le système permettra alors de déceler les variations significatives dans les «normes d'admission et de séjour".

Remarquons enfin que ce système, orienté vers la prévision, n'a pas été perçu par les membres du personnel infirmier comme un nouveau moyen inventé pour les surveiller dans l'exercice de leurs fonctions, mais plutôt comme un système qui les aide à mieux anticiper les besoins des patients traités.

Le système nécessite encore de nombreuses améliorations. En particulier nous devons éliminer l'hypothèse sous-jacente de stationnarité des processus 
TABLEAU V

Réduction de la variance par les données recueillies à thôpital.

\begin{tabular}{|c|c|c|c|c|}
\hline & & \multicolumn{3}{|c|}{ QUART } \\
\hline & & Jour & Soir & Nuit \\
\hline \multicolumn{2}{|c|}{ Variance originale } & \multirow{2}{*}{18263.66} & \multirow{2}{*}{13499.42} & \multirow{2}{*}{5752.16} \\
\hline Jour & Statistique & & & \\
\hline 1 & $\begin{array}{c}\text { Variance } \\
\gamma\end{array}$ & $\begin{array}{c}4164.74 \\
.76\end{array}$ & $\begin{array}{c}2650.04 \\
.80\end{array}$ & $\begin{array}{c}1174.56 \\
.79\end{array}$ \\
\hline 2 & $\begin{array}{c}\text { Variance } \\
\gamma\end{array}$ & $\begin{array}{c}4751.95 \\
.73\end{array}$ & $\begin{array}{c}3651.09 \\
.72\end{array}$ & $\begin{array}{c}2179.78 \\
.61\end{array}$ \\
\hline 3 & $\begin{array}{c}\text { Variance } \\
\gamma\end{array}$ & $\begin{array}{c}8292.37 \\
.53\end{array}$ & $\begin{array}{c}6952.83 \\
.46\end{array}$ & $\begin{array}{c}4388.81 \\
.21\end{array}$ \\
\hline 4 & $\begin{array}{c}\text { Variance } \\
\gamma\end{array}$ & $\begin{array}{c}12214.87 \\
.30\end{array}$ & $\begin{array}{c}9976.53 \\
.23\end{array}$ & $\underset{* * *}{6277.20}$ \\
\hline 5 & $\begin{array}{c}\text { Variance } \\
\gamma\end{array}$ & $\underset{* * *}{17862.98}$ & $\underset{* * *}{13799} \cdot 41$ & $\underset{* * *}{8014} .61$ \\
\hline 6 & $\begin{array}{c}\text { Variance } \\
\gamma\end{array}$ & $21 \underset{* * *}{349.50}$ & $\underset{* * *}{16125.90}$ & $\underset{* * *}{8863 .} 20$ \\
\hline 7 & $\begin{array}{c}\text { Variance } \\
\gamma\end{array}$ & $\underset{* * *}{24744.24}$ & $\underset{* * *}{17983} .11$ & $\underset{* * *}{9486.99}$ \\
\hline
\end{tabular}

décrivant la dynamique des maladies et la remplacer par une description périodique tenant compte en particulier de l'effet des fins de semaines où peu de patients sont admis ou opérés. Cette modification est théoriquement facile à réaliser mais se heurte très vite à des contraintes de capacité et de temps de traitement.

Le système doit d'autre part être complété par un ensemble de modèles périphériques dédiés à la gestion du personnel ou à l'ordonnancement des opérations et qui pourraient s'alimenter aux prévisions réalisées par le système.

\section{REMERCIEMENTS}

Cette recherche a pu être réalisée grâce à la collaboration de nombreuses personnes à l'hôpital général juif de Montréal. En particulier M. H. Elbaz, les Drs P. Heilpern et J. Shindler, Garde A. Layola ont pris une part active dans l'expérience pilote.

D'autres chercheurs du GERAD ont été associés à divers stades de cette recherche. Daniel Dollo, Denis Berthold, Marie Robillard et Krista Frénois ont participé à la programmation ou au recueil des données. 


\section{BIBLIOGRAPHIE}

1. F. Chauny, M. Chokron et A. Haurie, Nursing Care Demand Prediction Based on a Decomposed Semi-Markov Population Model, Operations Research Letters, mars 1984, vol. $2, \mathrm{n}^{\circ} 6$, p. 279-284.

2. M. Chokron et al., P.S.I., Prévision de la demande de soins infirmiers. Manuel d'utilisation, les Cahiers du GERAD, G-81-01, 1981.

3. M. Chokron et al., P.S.I., A Computerized Information System to Forecast the Nursing Care Demand. User's Guide, les Cahiers du GERAD, G-81-02, 1981.

4. M. Chokron et A. Haurie, Prévision et régulation de la demande de soins infirmiers à partir d'un système d'information autonome, Third Congress of the European Federation of Medical Informatics Proceedings, Toulouse, France, mars 1981.

5. D. Collart et A. Haurie, On a Suboptimal Control of a Hospital Iñàtient Admission System, I.E.E.E. Transactions on Automatic Control, avril 1976, p. 233-238.

6. D. Collard et A. Haurie, On the Control of Care Supply and Demand in a Urology Department, European Journal of Operations Research, vol. $4, \mathrm{n}^{\circ} 3$, 1980, p. $160-172$.

7. J. P. Dion et D. Gautier, Estimation des paramètres du processus semi-Markovien de l'évolution intra-hospitalière des coronariens, Actes du Colloque sur la Théorie des Systèmes et de la Gestion Scientifique des Services Publics, C.R.M., Université de Montréal, École des H.E.C., 1975.

8. J. C. Hershey, E. N. Weiss et M. A. Cohen, A Stochastic Service Network Model with Application to Hospital Facilities, Operations Research, vol. 29, 1981, p. 1-22.

9. D. C. Holloway et D. M. Warner, Decision Making and Control for Health Administration, Health Administration Press, University of Michigan, 1978.

10. E. P. C. KAO, A Semi-Markov Model to Predict Recovery Progress of Coronary Patients, Health Services Research, 1971, p. 191-208.

11. E. P. C. KAo, A Semi-Markov Population Model with Application to Hospital Planning, I.E.E.E. Transaction on Systems Man and Cybernetics, vol. 4, 1973, SMC-3.

12. P. Kolesar, A Markovian Model for Admission Scheduling, Management Science, vol. 16, n 6, février 1970.

13. F. L. Offensend, A Hospital Admission System Based on Nursing Workload, Management Science, vol. 19, $\mathrm{n}^{\circ}$ 2, octobre 1972.

14. R. C. Smallwood, G. E. Murray, D. D. Sylva, E. J. Sovdik et L. M. Klainer, A Medical Requirements Model for Health System Design, Proceedings of I.E.E.E., vol. 57, (11), 1969, p. 1880-1887.

15. C. Tilquin, PRN 76, un système d'information pour la gestion des soins infirmiers, Rapport de Recherche, EROS, Université de Montréal, 1978.

16. D. M. WARner, Scheduling Nursing Personnel According to Nursing Preference: A Mathematical Programming Approach, Operations Research, vol. 24, $\mathrm{n}^{\circ} 5$, septembre-octobre 1976 , p. 842-856.

17. E. N. Weiss, M. A. Cohen et J. C. Hershey, An Iterative Estimation and Validation Procedure for Specification of Semi-Markov Models with Application to Hospital Patient Flow, Operations Research, vol. 30, 1982, p. 1082-1104.

18. H. Wolfe et J. P. Young, Staffing the Nursing Unit; Part I: Nursing Research, vol. 14, $\mathrm{n}^{\circ}$ 3, été 1965; Part II: Nursing Research, vol. 14, $\mathrm{n}^{\circ} 4$, automne 1965. 


\section{ANNEXE}

Le but de la simulation est de produire, à partir du recensement de l'unité, pour un jour, le recensement du lendemain. Le diagramme suivant illustre la procédure utilisée.

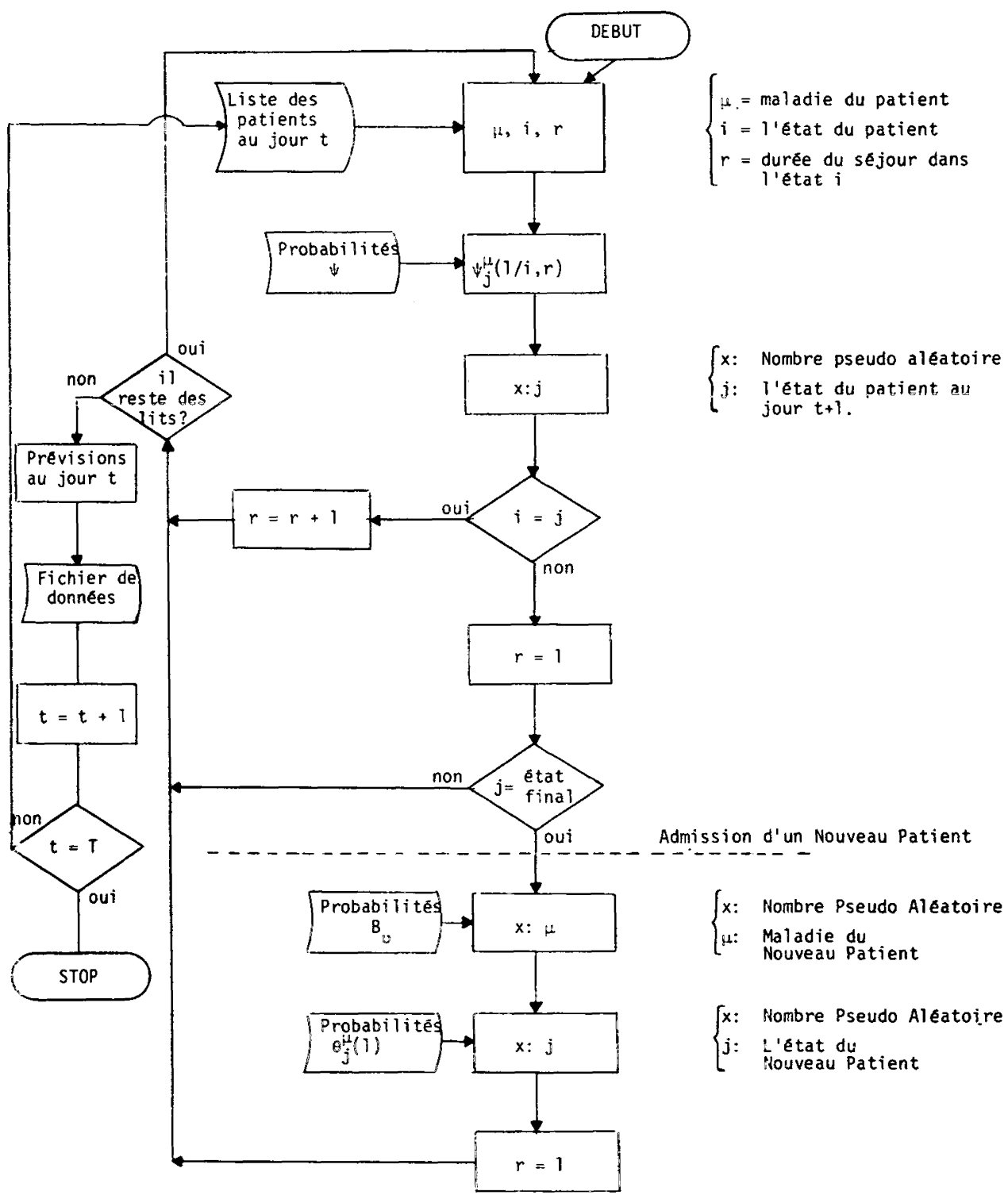

vol. $18, \mathrm{n}^{\circ} 2$, mai 1984 